\title{
Hipercalcemia e lesões osteolíticas múltiplas em criança com paracoccidioidomicose disseminada e tuberculose pulmonar
}

\author{
Hypercalcemia and multiple osteolytic lesions in a child \\ with disseminated paracoccidioidomycosis and pulmonary tuberculosis \\ Antonia T. Tresoldi ${ }^{1}$, Ricardo M. Pereira2 ${ }^{2}$, Lelma C. Castro ${ }^{3}$, \\ Sumara Z. P. Rigatto ${ }^{4}$, Vera M. S. Belangero ${ }^{1}$
}

\section{Resumo}

Objetivo: Descrever o caso de uma criança com paracoccidioidomicose, que apresentou hipercalcemia associada a múltiplas lesões osteolíticas.

Descrição: Menino de 6 anos, internado com história de febre e hepatoesplenomegalia há 1 mês. À internação, apresentava-se em regular estado geral, descorado, com linfonodomegalia generalizada e hepatoesplenomegalia. Os exames laboratoriais identificaram anemia (hemoglobina $=6,8 \mathrm{~g} / \mathrm{dl}$ ), eosinofilia $\left(1.222 / \mathrm{mm}^{3}\right)$, plaquetopenia $\left(102.000 / \mathrm{mm}^{3}\right.$ ) e hipoalbuminemia (albumina $=2,2 \mathrm{~g} / \mathrm{dl}$ ). Paracoccidioides brasiliensis foi identificado no mielograma. A partir da segunda semana de internação, apresentou artralgia, hipoatividade e dificuldade à deambulação, sendo constatada hipercalcemia (dosagem máxima de $14,9 \mathrm{mg} \%$ ) e redução da função renal, que duraram pouco mais de 2 semanas. No $42^{\circ}$ dia de internação, foram vistas, na radiografia de tórax, múltiplas lesões líticas em clavículas, escápulas, costelas e úmeros, com escorregamento epifisário de úmero bilateral. Apresentou nefrocalcinose e nefrolitíase, com redução no clearance de creatinina e evidências de lesão tubular. No final do segundo mês de internação, na cultura do lavado gástrico, foi identificado Mycobacterium tuberculosis. Recebeu tratamento para paracoccidioidomicose e tuberculose e está há mais de 3 anos em acompanhamento, sem nenhuma seqüela.

Comentários: $O$ desenvolvimento da hipercalcemia sintomática, levando à lesão renal e associada a lesões ósseas múltiplas e disseminadas, nunca foi descrito em paracoccidioidomicose. Embora tenha sido diagnosticada tuberculose pulmonar, que pode estar relacionada à hipercalcemia, a forma abrupta como se instalou e como se normalizou sem o tratamento específico para tuberculose sugere que a lise óssea foi o fator mais importante na gênese da hipercalcemia.

J Pediatr (Rio J). 2005;81(4):349-52: Paracoccidioidomicose, tuberculose, hipercalcemia, lesões osteolíticas, crianças.

\begin{abstract}
Objective: To describe the case of a child with paracoccidioidomycosis who presented hypercalcemia with multiple osteolytic lesions.

Description: A 6-year-old boy was admitted with a one-month history of fever and hepatosplenomegaly. On admission, he looked sick, pale, and had disseminated lymphadenopathy and hepatosplenomegaly. The laboratory findings included anemia (hemoglobin $=6.8 \mathrm{~g} / \mathrm{dl}$ ), eosinophilia $\left(1,222 / \mathrm{mm}^{3}\right)$, thrombocytopenia $\left(102,000 / \mathrm{mm}^{3}\right)$, and hypoalbuminemia (serum albumin $=2.2 \mathrm{~g} / \mathrm{dl}$ ). Paracoccidioides brasiliensis was identified in bone marrow examination. In the second week after admission, the patient presented joint pain, poor activity and difficulty in walking. He presented hypercalcemia (maximum value $=$ $14.9 \mathrm{mg} \%$ ) and reduction in renal function, which lasted for two weeks. On the 42 nd day after admission, his chest X-ray showed lytic lesions in clavicle, scapula, ribs, and humerus, with bilateral slipped capital humeral epiphysis. The patient presented nephrocalcinosis and nephrolithiasis, reduction in creatinine clearance and evidence of tubular lesions. At the end of the second month after admission, Mycobacterium tuberculosis was isolated in gastric lavage. The child received treatment for paracoccidioidomycosis and tuberculosis and has not had any sequelae for 3 years.

Comments: The development of symptomatic hypercalcemia leading to renal lesion, associated with multiple osteolytic lesions, had never been described in paracoccidioidomycosis. Although pulmonary tuberculosis was diagnosed and could be related to hypercalcemia, the sudden onset of hypercalcemia and its normalization without specific treatment for tuberculosis suggests that bone lysis was the most important factor in the genesis of hypercalcemia.
\end{abstract}

J Pediatr (Rio J). 2005;81(4):349-52: Paracoccidioidomycosis, tuberculosis, hypercalcemia, osteolytic lesions, children.

1. Professora associada, Departamento de Pediatria, Faculdade de Ciências Médicas, Universidade Estadual de Campinas (UNICAMP), Campinas, SP.

2. Doutor. Médico assistente, Departamento de Pediatria, Faculdade de Ciências Médicas, UNICAMP, Campinas, SP.

3. Ex-residente, Departamento de Pediatria, Faculdade de Ciências Médicas, UNICAMP, Campinas, SP.

4. Doutora. Professora, Departamento de Pediatria, Faculdade de Ciências Médicas, UNICAMP, Campinas, SP.

Artigo submetido em 03.03.05, aceito em 13.05.05.

Como citar este artigo: Tresoldi AT, Pereira RM, Castro LC, Rigatto SZ, Belangero VM. Hipercalcemia e lesões osteolíticas múltiplas em criança com paracoccidioidomicose disseminada e tuberculose pulmonar. J Pediatr (Rio J). 2005;81:349-52. 


\section{Introdução}

A paracoccidioidomicose (PCM) é uma doença fúngica causada pelo Paracoccidioides brasiliensis. Apresenta-se nas formas subaguda ou juvenil e na forma crônica ou tipo adulto. Na forma crônica, o órgão mais acometido é o pulmão. Já na forma juvenil, que acomete crianças e adultos jovens, a principal manifestação clínica é a de uma síndrome linfoproliferativa febril ${ }^{1-5}$. Nessa apresentação clínica, o acometimento osteoarticular ocorre em até $20 \%$ dos casos, com lesões líticas atingindo qualquer osso, mas predominando no tórax e cintura escapular 6,7 .

A hipercalcemia já foi descrita em doenças granulomatosas como sarcoidose e tuberculose ${ }^{8-10}$, bem como em doenças fúngicas ${ }^{11}$, mas raramente associada à $\mathrm{PCM}^{12}$. Desse modo, o objetivo deste trabalho é descrever o caso de uma criança com PCM que apresentou hipercalcemia e lesões osteolíticas difusas.

\section{Descrição do caso}

Menino de 6 anos de idade, branco, internou com história de febre alta há um mês, evoluindo com aumento de volume abdominal e lesões na pele 3 dias após. Veio encaminhado do serviço básico de saúde por não apresentar melhora da febre e por ter sido evidenciada hepatoesplenomegalia.

Apresentava-se em regular estado geral, descorado, com linfonodos pequenos ( 1,0 a 1,5 cm) e móveis, palpáveis em todas as cadeias cervicais e axilares. Apresentava lesões cutâneas (variceliformes) na face e tronco. $\mathrm{O}$ abdome era globoso, tenso, com fígado palpado a $8 \mathrm{~cm}$ do rebordo costal direito, endurecido, e baço palpado a $10 \mathrm{~cm}$ do rebordo costal esquerdo, endurecido. À internação, os exames laboratoriais mostravam: hemoglobina $6,8 \mathrm{~g} / \mathrm{dl}$; hematócrito $19,8 \%$; leucócitos $6.060 / \mathrm{mm}^{3}$ (56\% segmentados e $20 \%$ eosinófilos); plaquetas $102.000 / \mathrm{mm}^{3}$; aspartato aminotransferase $7 \mathrm{U} / \mathrm{l}$; alanina aminotransferase $27 \mathrm{U} / \mathrm{l}$; uréia $27 \mathrm{mg} / \mathrm{dl}$; creatinina 0,48 mg/dl; fosfatase alcalina $429 \mathrm{U} / \mathrm{l}$ (até $269 \mathrm{U} / \mathrm{l}$ ); gama glutamiltransferase $116 \mathrm{U} / \mathrm{l}(9-40 \mathrm{U} / \mathrm{l})$; albumina sérica $2,2 \mathrm{~g} / \mathrm{dl}(3,5-5 \mathrm{~g} / \mathrm{dl}) \mathrm{e}$ gamaglobulina sérica $2,72 \mathrm{~g} / \mathrm{dl}(0,8-1,6 \mathrm{~g} / \mathrm{dl})$. Pela suspeita de leucose, foi realizado mielograma, evidenciando grande quantidade de estruturas ovaladas com membrana birrefringente e com múltiplos brotamentos, sugerindo Paracoccidioides brasiliensis. A ultra-sonografia abdominal evidenciou fígado com ecotextura homogênea, dimensões aumentadas, chegando à fossa ilíaca e conglomerado de linfonodos no hilo hepático; baço de dimensões aumentadas $(16 \mathrm{~cm})$. A radiografia de tórax mostrou condensação peri-hilar e esqueleto normal. Com a hipótese de PCM, foi iniciado tratamento com sulfametoxazol-trimetoprima intravenoso ( $8 \mathrm{mg} / \mathrm{kg} /$ dia de trimetoprima). A sorologia para HIV foi negativa. Não foi realizada sorologia para PCM.

No final da primeira semana de internação, apresentava leucopenia (2.950 leucócitos $/ \mathrm{mm}^{3}$ ) e plaquetopenia $\left(48.000 / \mathrm{mm}^{3}\right)$, sendo então associada anfotericina $B$ desoxicolato (1 mg/kg/dia) ao tratamento inicial. No $14^{\circ}$ dia de internação, iniciou quadro de artralgia, hipoativi- dade e dificuldade à deambulação. Havia melhora da leucopenia (6.140 leucócitos $/ \mathrm{mm}^{3}$ ), com redução maior da albuminemia $(1,52 \mathrm{~g} / \mathrm{dl})$ e hipercalcemia $(11,2 \mathrm{mg} \%)$. No $20^{\circ}$ dia de internação, acentuou-se o distúrbio eletrolítico, apresentando calcemia de $12,9 \mathrm{mg} \%$, potassemia de $2,2 \mathrm{mEq} / \mathrm{l}$ e magnesemia de $1,8 \mathrm{mEq} / \mathrm{l}$. Ao exame físico, apresentava diminuição da hepatoesplenomegalia, sendo suspensa a anfotericina $B$, pensando-se num agravamento do distúrbio eletrolítico pela droga. No $25^{\circ}$ dia de internação, além da hipercalcemia ( 14,9 mg\%) e hipopotassemia $(2,2 \mathrm{mEq} / \mathrm{l})$ mantidas, apesar das medidas adotadas (hiper-hidratação intravenosa com solução salina, reposição de potássio e uso de furosemida $2 \mathrm{mg} / \mathrm{kg} / \mathrm{dia})$, apresentou elevação de uréia (62 mg/dl) e creatinina $(0,7 \mathrm{mg} / \mathrm{dl})$, com clearance de creatinina de 58 $\mathrm{ml} / \mathrm{min} / 1,72 \mathrm{~m}^{2}$. A dosagem de paratormônio foi $<1 \mathrm{pg} / \mathrm{ml}$ (normal de 12-72); a dosagem de fósforo sérico foi de $6,1 \mathrm{mg} / \mathrm{l}$ (normal de 3,5-5); a tubular máxima de fósforo (TmP) foi de $5 \mathrm{mg} / \mathrm{ml} / \mathrm{RFG}$ (normal de 2,5-4); calciúria de $13 \mathrm{mg} / \mathrm{kg} /$ dia (normal até 4); relação cálcio urinário/ creatinina urinária de 0,7 (normal até 0,21 ). Manteve quadro de dor nos membros e hipercalcemia até o $32^{\circ}$ dia de internação. Nesse momento, a ultra-sonografia abdominal evidenciou nefropatia parenquimatosa aguda, com nefrocalcinose bilateral e nefrolitíase à esquerda. Por manter quadro de apatia e hipoatividade, com a radiografia de tórax ainda evidenciando condensação peri-hilar, sugerindo linfonodomegalia, foi feita coleta de lavado gástrico para pesquisa de tuberculose e realizada biópsia hepática para investigação de outra doença que pudesse estar relacionada com a evolução não favorável. Foi também realizado novo mielograma, que mostrou hipoplasia acentuada e ausência de parasitas.

No $42^{\circ}$ dia de internação, a radiografia de tórax evidenciou, pela primeira vez, lesões líticas de clavículas, escápulas, costelas e úmeros; escorregamento epifisário de úmero bilateral e fratura de úmero esquerdo (Figuras 1,2 e 3).

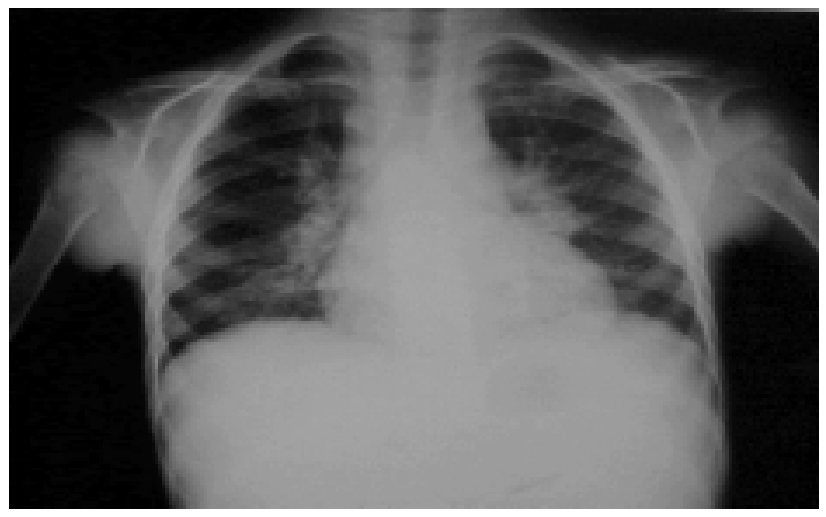

Figura 1 - Radiografia de tórax mostrando condensação perihilar e lesões líticas em clavículas, escápulas e úmeros; escorregamento epifisário de úmero bilateral e fratura de úmero esquerdo 
A cintilografia óssea com MDP-99mTc mostrou hipercaptação difusa na calota craniana, arcos costais anteriores e posteriores, úmeros, terço proximal dos rádios, ossos da bacia e fêmures. Como a biópsia hepática dessa época evidenciava ainda grande quantidade de fungos, foi reiniciada a anfotericina $B$, que foi utilizada por mais 20 dias. Nesse período, houve normalização do clearance de creatinina ( $\left.86 \mathrm{ml} / \mathrm{min} / 1,72 \mathrm{~m}^{2}\right)$, mas a relação alfa 1 microglobulina/creatinina urinária era de 10,64 (normal até 0,17$)$, sugerindo lesão tubular. Na alta, essa relação era de 0,44 .

No final do segundo mês de internação, na cultura do lavado gástrico, foi identificado Mycobacterium tuberculo-

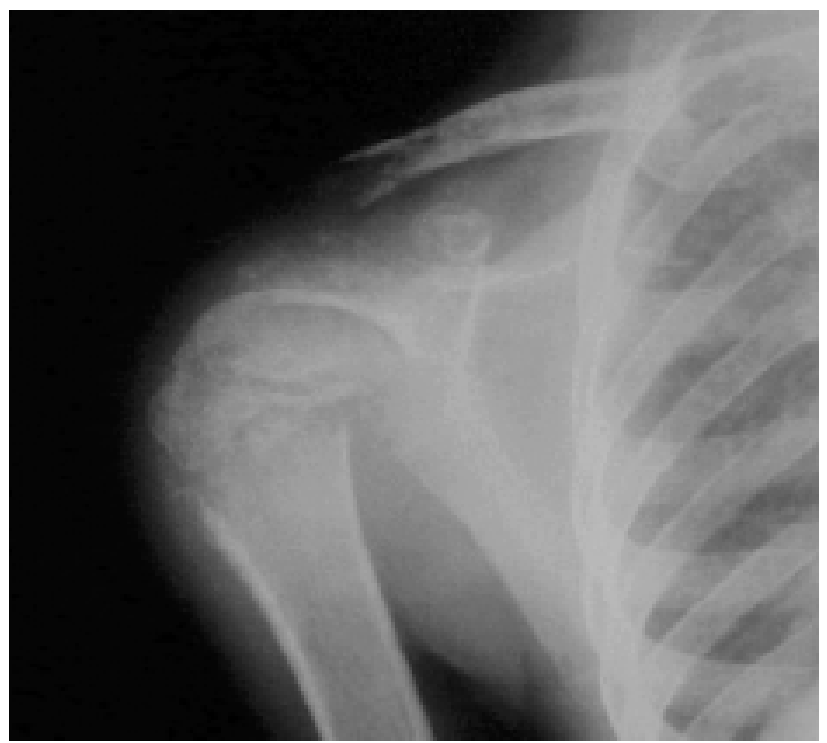

Figura 2 - Detalhes de radiografias do ombro direito

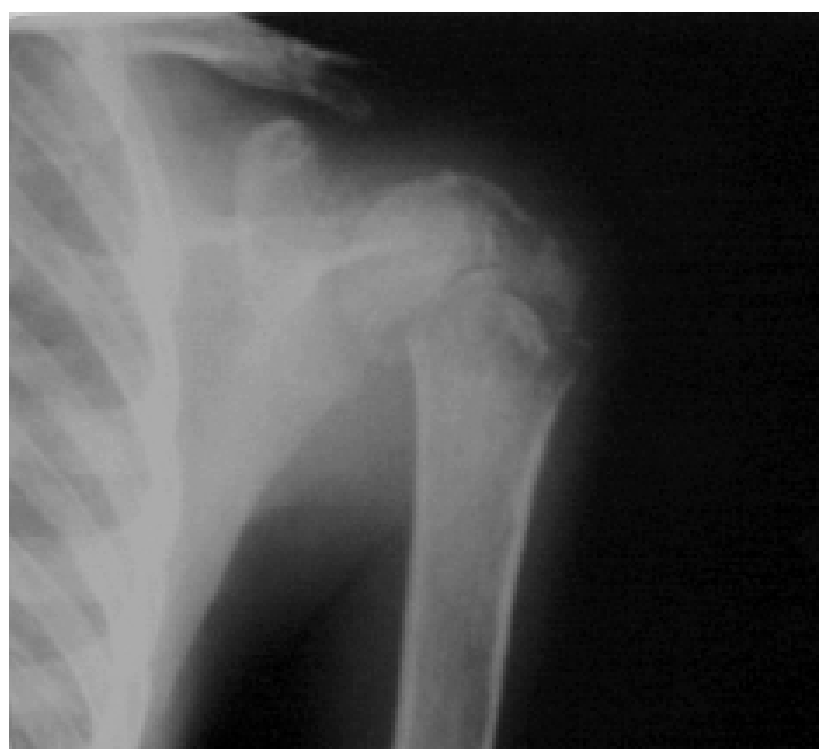

Figura 3 - Detalhes de radiografias do ombro esquerdo sis, sendo iniciado esquema tríplice com isoniazida, rifampicina e pirazinamida. O acometimento ósseo foi conduzido de forma conservadora, apenas com imobilização da fratura do úmero.

Ao completar 3 meses de internação, o paciente recebeu alta, apresentando limitação de abdução dos braços, recebendo sulfametoxazol-trimetoprima via oral e esquema tríplice para tuberculose. Atualmente, está há mais de 3 anos em acompanhamento ambulatorial, já completou o tratamento programado, apresenta normalização completa dos parâmetros laboratoriais e mobilidade normal das articulações do ombro.

Foi obtido o consentimento livre e esclarecido, por escrito, da responsável pelo paciente.

\section{Comentários}

A PCM na criança apresenta-se com envolvimento predominante do sistema reticuloendotelial, caracterizado como uma síndrome linfoproliferativa febril. Alguns autores sugerem que as crianças maiores de 10 anos tendem a ter maior envolvimento osteoarticular e cutâneo do que as crianças menores, nas quais o fígado e o baço são mais freqüentemente envolvidos ${ }^{1,2,4,5}$. A apresentação clínica do caso aqui descrito foi de uma síndrome linfoproliferativa febril com envolvimento ósseo.

As alterações laboratoriais são inespecíficas, como anemia, eosinofilia, hipoalbuminemia, hipergamaglobulinemia e aumento da fosfatase alcalina e da velocidade de hemossedimentação 4,7 . No caso, as alterações laboratoriais iniciais não demonstraram comprometimento hepático ou renal. Apesar da biópsia hepática ter demonstrado grande quantidade de fungos, não houve evidência laboratorial de lesão hepatocelular, havendo apenas elevação da gama glutamiltransferase, que pode ser explicada pela compressão das vias biliares por linfonodos do hilo hepático. Outros autores já relataram essa situação, geralmente associada à hiperbilirrubinemia13-15.

A hipoplasia de medula óssea, a qual persistiu por mais de um mês após o início do tratamento, provavelmente foi causada pela infecção fúngica, demonstrada no primeiro mielograma, e não teve relação com o uso de anfotericina B ou da sulfonamida, já que houve recuperação da medula apesar da manutenção dessas drogas.

O diagnóstico de tuberculose não foi uma ocorrência isolada no paciente aqui descrito. A associação das duas doenças granulomatosas é descrita em aproximadamente $10 \%$ dos casos de $\mathrm{PCM}^{16}$.

A hipercalcemia é definida como nível de cálcio sérico acima de $11 \mathrm{mg} \%$ ou nível de cálcio iônico maior que $5,6 \mathrm{mg} \%$ (ou $>1,4 \mathrm{mmol} / \mathrm{l})^{17}$. No $14^{\circ}$ dia da evolução do caso, o paciente iniciou queixa de artralgia e dificuldade à deambulação, associados a valores da calcemia de $11,2 \mathrm{mg} \%$ e de albuminemia de $1,5 \mathrm{~g} \%$. Como cada grama\% de redução de albuminemia reduz $0,8 \mathrm{mg} \%$ da calcemia, o maior valor que poderia ser esperado de calcemia, nesta circunstância, seria de $8 \mathrm{mg} \%{ }^{17}$, de- 
monstrando que, já nessa avaliação, a calcemia era bastante elevada. Tendo em conta a radiografia de tórax do $42^{\circ}$ dia de internação, onde se observavam múltiplas e extensas lesões osteolíticas, as quais não eram visíveis nas avaliações radiológicas anteriores, a principal hipótese para a hipercalcemia passou a ser a destruição óssea pelo fungo. A presença de hipercalciúria, avaliada em mg/ dia ou pela relação cálcio/creatinina urinária; a supressão do hormônio paratireoidiano (PTH); a hiperfosfatemia associada à tubular máxima de fosfato elevada18; o aparecimento de nefrocalcinose e nefrolitíase e a piora do ritmo de filtração glomerular, cronologicamente concomitantes à hipercalcemia, são todos efeitos compatíveis com o aumento da disponibilidade de cálcio e fósforo no líquido extracelular ${ }^{17-19}$. A falta de antecedente de uso de vitamina $D$ elimina também a possibilidade de intoxicação por vitamina $D$, que poderia se expressar com quadro laboratorial semelhante.

É interessante salientar que, provavelmente, ocorreu, neste caso, o efeito conhecido da "má" adaptação renal à hipercalcemia, isto é, a elevação da calciúria ocorre em proporção menor que o aumento da carga filtrada de cálcio, mantendo-se a calcemia elevada18,19. Embora esse efeito proteja relativamente o rim da deposição intratubular de cálcio, atua de forma a manter a hipercalcemia ${ }^{19}$.

Outros efeitos da hipercalcemia sobre o rim incluem também a piora da ação da vasopressina, vasoconstrição arteriolar e redução do coeficiente de ultrafiltração do capilar glomerular ${ }^{19}$. Embora a poliúria não tenha sido detectada, houve diminuição da função renal, que chegou a clearance de $58 \mathrm{ml} / \mathrm{min} / 1,72 \mathrm{~m}^{2}$. As medidas instituídas de hiper-hidratação e uso de furosemida foram suficientes para o restabelecimento rápido da calcemia e, em médio prazo, da função renal. A detecção da elevação e normalização da relação $\alpha 1$ microglobulina/creatinina urinária reforça que o efeito da lesão tubular tenha sido transitório e reversível.

Na literatura, há relatos de hipercalcemia associada a doenças granulomatosas, como a produzida pela tuberculose $^{9}$, sarcoidose ${ }^{10}$, histoplasmose ${ }^{11}$ e criptococose ${ }^{20}$, sendo encontrado apenas um caso descrito em $\mathrm{PCM}^{12}$. Não há, nesses relatos, associação com lesões osteolíticas, sendo a hipercalcemia pouco esclarecida. Especulase ser devido ao aumento de síntese da $1,25(\mathrm{OH})_{2} \mathrm{D}_{3}$, em macrófagos $9,10,21$. Entretanto, em coccidioidomicose, esse mecanismo já foi contestado 22 . A possibilidade de a hipercalcemia ser também, no caso descrito, em parte devido a mecanismo semelhante não é possível de ser afastada, tendo em vista a associação com tuberculose. No entanto, a forma abrupta como se instalou e como se normalizou, além de todas as correlações acima descritas, sugere que a lise óssea foi o fator mais importante na gênese da hipercalcemia.

\section{Referências}

1. Brummer E, Castaneda E, Restrepo A. Paracoccidioidomycosis: an update. Clin Microbiol Rev. 1993;6:89-117.

2. Bittencourt AL, Andrade JA, Filho SP. Paracoccidioidomycosis in a four-year-old boy. Mycopathologia. 1986;93:55-9.

3. Castro RM, Del Negro G. Particularidades clínicas da paracoccidioidomicose na criança. Rev Hosp Clin Fac Med. $1976 ; 31: 194-8$.

4. Hildebrando TM, Filho NA, Filho FQ, Costa O, Miasaki N, Mira JG, et al. Paracoccidioidomicose na criança. Aspectos clínicos e laboratoriais em 25 casos. J Pediatr. 1987;63:92-7.

5. Londero AT, Melo IS. Paracoccidiodomycosis in childhood - a critical review. Mycopathologia. 1983;82:49-55.

6. Silva AF. Contribuição aos estudos da paracoccidioidomicose no Paraná e das lesões ósseas e articulares paracoccidióidicas [tese de livre docência]. Curitiba (PR): Universidade Federal do Paraná; 1976.

7. Pereira RM, Bucaretchi F, Barison EM, Tresoldi AT. Paracoccidioidomycosis in children: clinical presentation, followup and outcome. Rev Inst Med Trop S. Paulo. 2004;46:127-31.

8. Adams JS, Gacad MA. Characterization of $1 \alpha$ hydroxylation of vitamin D3 sterols by cultured alveolar macrophages from patients with sarcoidosis. J Exp Med. 1985;161:755-65.

9. Abbasi AA, Chemplavil JK, Muller BF, Arnstein AR. Hypercalcemia in active pulmonary tuberculosis. Ann Intern Med. 1979;90: 324-8.

10. Bilezikian JP. Etiologies and therapy of hypercalcemia. Endocrinol Metab Clin North Am. 1989;18:389-414.

11. Murray JJ, Heim CR. Hypercalcemia in disseminated histoplasmosis. Am J Med. 1985;78:881-4.

12. Silva LC, Ferrari TC. Hypercalcaemia and paracoccidioidomycosis. Trans R Soc Trop Med Hyg. 1998;92:187.

13. Daher RR, Wasconcelos WM, Cardoso VM. Fígado e blastomicose sul-americana. J Bras Med. 1973;25:83-90.

14. Nahomi N. Icterícia obstrutiva na doença de Lutz. A propósito de dois casos. Hospital (Rio de Janeiro). 1962;62:1243-54.

15. Pinto WP. Contribuição ao estudo do comprometimento hepático na paracoccidioidomicose [dissertação]. São Paulo (SP): Universidade de São Paulo; 1980.

16. Del Negro G. Outras lesões. Formas de ocorrência rara e associações com outros processos. In: Del Negro G, Lacaz CS, Fiorelo AM, editores. Paracoccidioidomicose - blastomicose sulamericana. São Paulo: Savier - Edusp; 1982. p. 229-43.

17. Harrison HE, Harrison HC. Calcium and phosphate homeostasis. In: Harrison HE, Harrison HC, editors. Disorders of calcium and phosphate metabolism in childhood and adolescence. 1st ed. Philadelphia: W. B. Saunders; 1979. p. 15-46.

18. Kanis JA, Handy MA. Hypo-Hypercalcemia. In: Oxford Textbook of Clinical Nephrology. 2nd ed. Oxford: Oxford University Press; 1998. p. 225-49.

19. Harrison HE, Harrison HC. Hypercalcemic states. In: Harrison $\mathrm{HE}$, Harrison HC, editors. Disorders of calcium and phosphate metabolism in childhood and adolescence. 1st ed. Philadelphia: W. B. Saunders; 1979. p. 100-140.

20. Ali MY, Gopal KV, Llerena LA, Taylor HC. Hypercalcemia associated with infection by Criptococcus neoformans and Coccidioides immitis. Am J Med Sci. 1999;318:419-23.

21. Lawn SD, Macallan DC. Hypercalcemia: a manifestation of immune reconstitution complicating tuberculosis in an HIVinfected person. Clin Infect Dis. 2004;38:154-5.

22. Caldwell JW, Arsura EL, Kilgore WB, Reddy CM, Johnson RH. Hypercalcemia in patients with disseminated coccidioidomycosis. Am J Med Sci. 2004;327:15-8.

Correspondência:

Antonia Teresinha Tresoldi

Rua José Paulino, 1875/43 A

CEP 13013-002 - Campinas, SP

E-mail: tresoldi@hc.unicamp.br 